\title{
SUPPORTING INFORMATION - Patterned Cellulose Nanocrystal Aerogel Films with Tunable Dimensions and Morphologies as Ultra-Porous Scaffolds for Cell Culture
}

Tyler Or, ${ }^{\dagger}$ Sokunthearath Saem, ${ }^{\dagger}$ Aurore Esteve, ${ }^{\dagger}$ Daniel A. Osorio,${ }^{\S}$ Kevin J. De France, ${ }^{\S}$ Jaana Vapaavuori, ${ }^{\ddagger}$ Todd Hoare, ${ }^{\S}$ Aline Cerf, Emily D. Cranston, ${ }^{\S}$ and Jose M. Moran-Mirabal ${ }^{\dagger *}$

'Department of Chemistry and Chemical Biology, McMaster University, 1280 Main St. West, Hamilton, Ontario L8S 4M1, Canada.

"LAAS-CNRS, Université de Toulouse, CNRS, Toulouse 31400, France

${ }^{\S}$ Department of Chemical Engineering, McMaster University, Hamilton, ON, L8S 4M1, Canada.

${ }^{\mathfrak{f}}$ Départment de Chimie, Université de Montréal, C.P. 6128, Succursale Centre-Ville, Montréal, QC, H3C 3J7E, Canada.

\section{KEYWORDS}

Micropatterning, freeze casting, microporous materials, hierarchically porous, flexible, CNC, POEGMA, cell scaffold.

Corresponding author: Prof. Jose Moran-Mirabal, mirabj@mcmaster.ca 

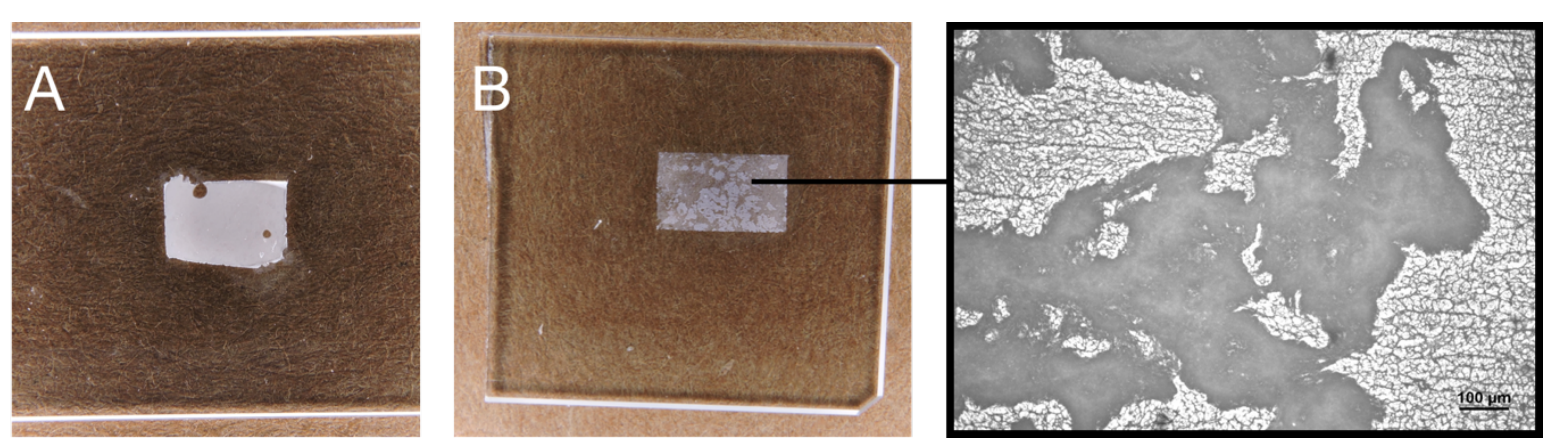

Figure S1. Optimization of the aerogel casting method. A) The presence of 'holes' in the aerogel was frequently seen when organic residue or surfactant on the substrate were not removed entirely. B) Areas of aggregation appeared less translucent relative to their surroundings. This occurred when the gel was cast several minutes after pre-mixing the aldehyde and hydrazide solutions or when the gel was frozen at $\geq-20^{\circ} \mathrm{C}$.
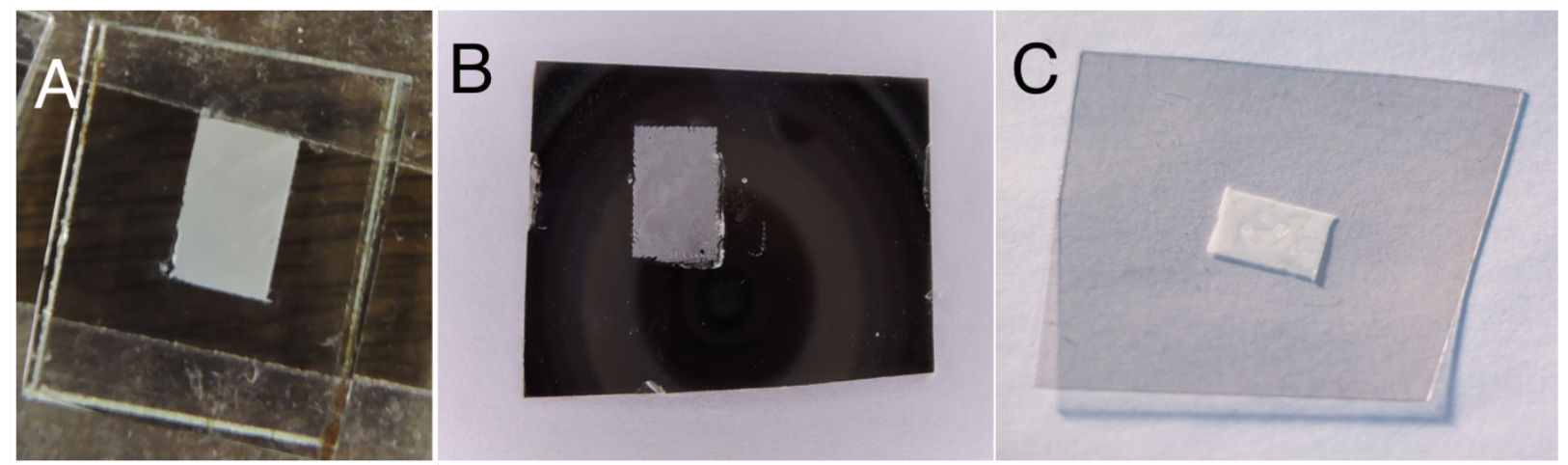

Figure S2. CNC-POEGMA aerogel film cast on various substrates. A) FTO-coated glass substrate. Aerogel fabricated using $21 \mu \mathrm{m}$-thick Parylene mold. B) Pt-coated PET substrate. Aerogel fabricated using $7 \mu \mathrm{m}$-thick Parylene mold. C) ITO-coated PET substrate. Aerogel fabricated using $85 \mu \mathrm{m}$-thick adhesive mold. 


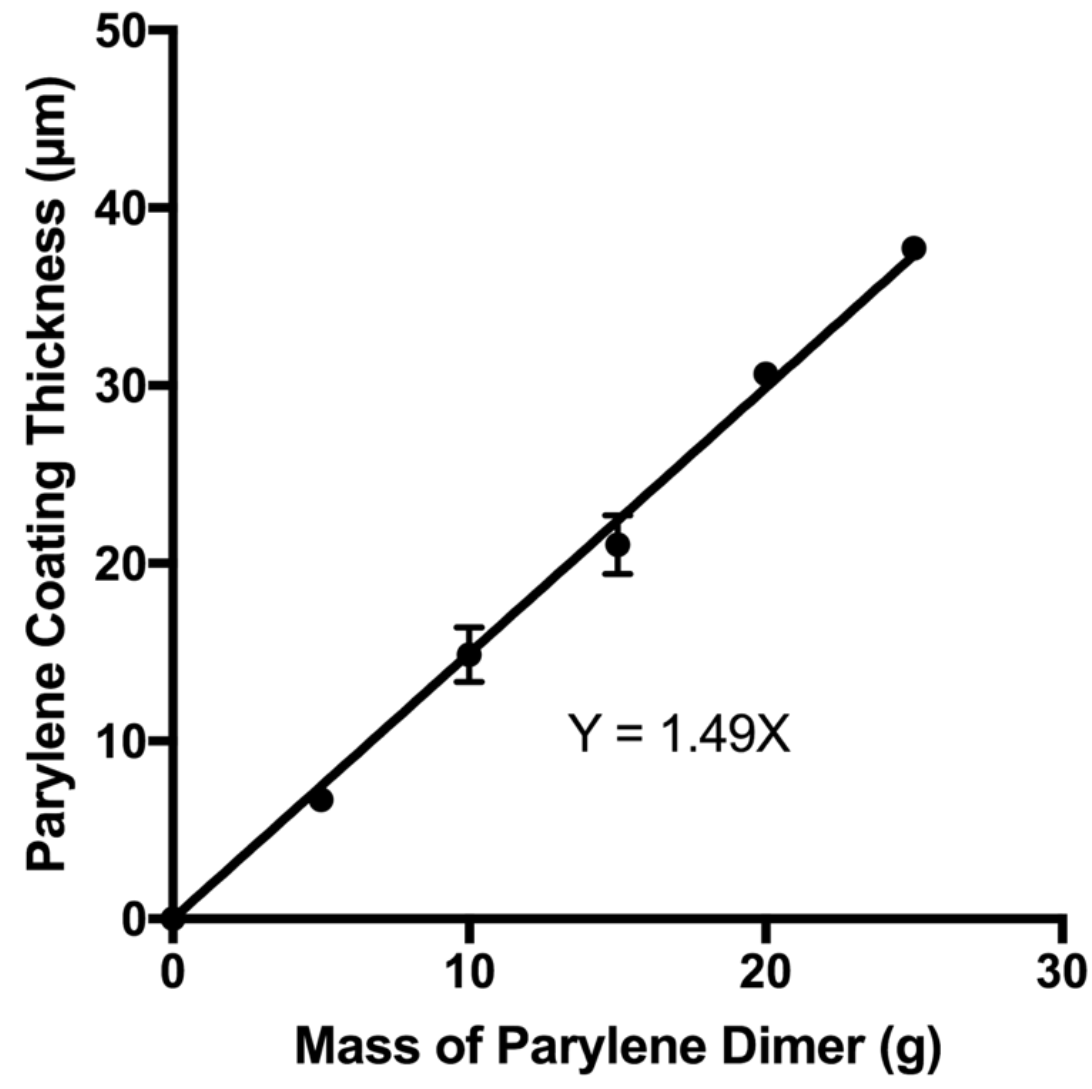

Figure S3. Parylene coating thickness increases linearly with respect to the amount of dimer loaded. 


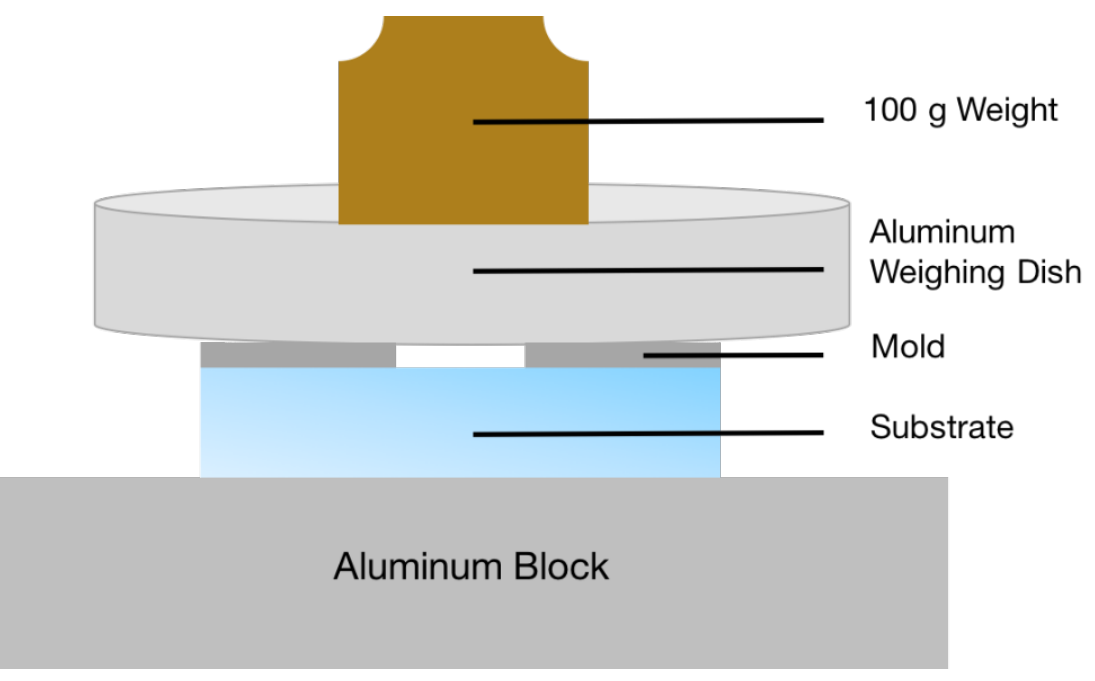

Figure S4. Pressure-applying setup.

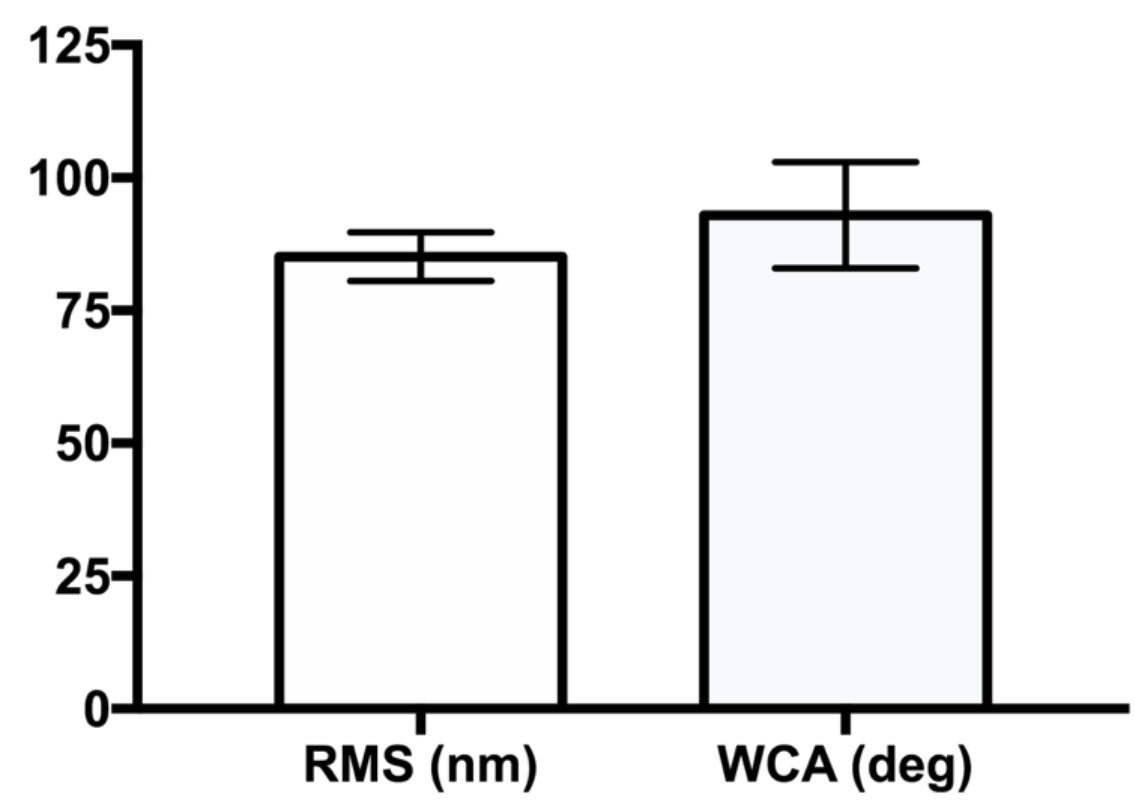

Figure S5. Average ( \pm standard deviation, $n \geq 3$ ) surface characteristics of aluminum weighing dishes used as the material in contact with the gel. Root mean square (RMS) surface roughness was measured by atomic force microscopy using $20 \times 20 \mu \mathrm{m}$ images. Water contact angle (WCA) indicates the wettability of the surface. 


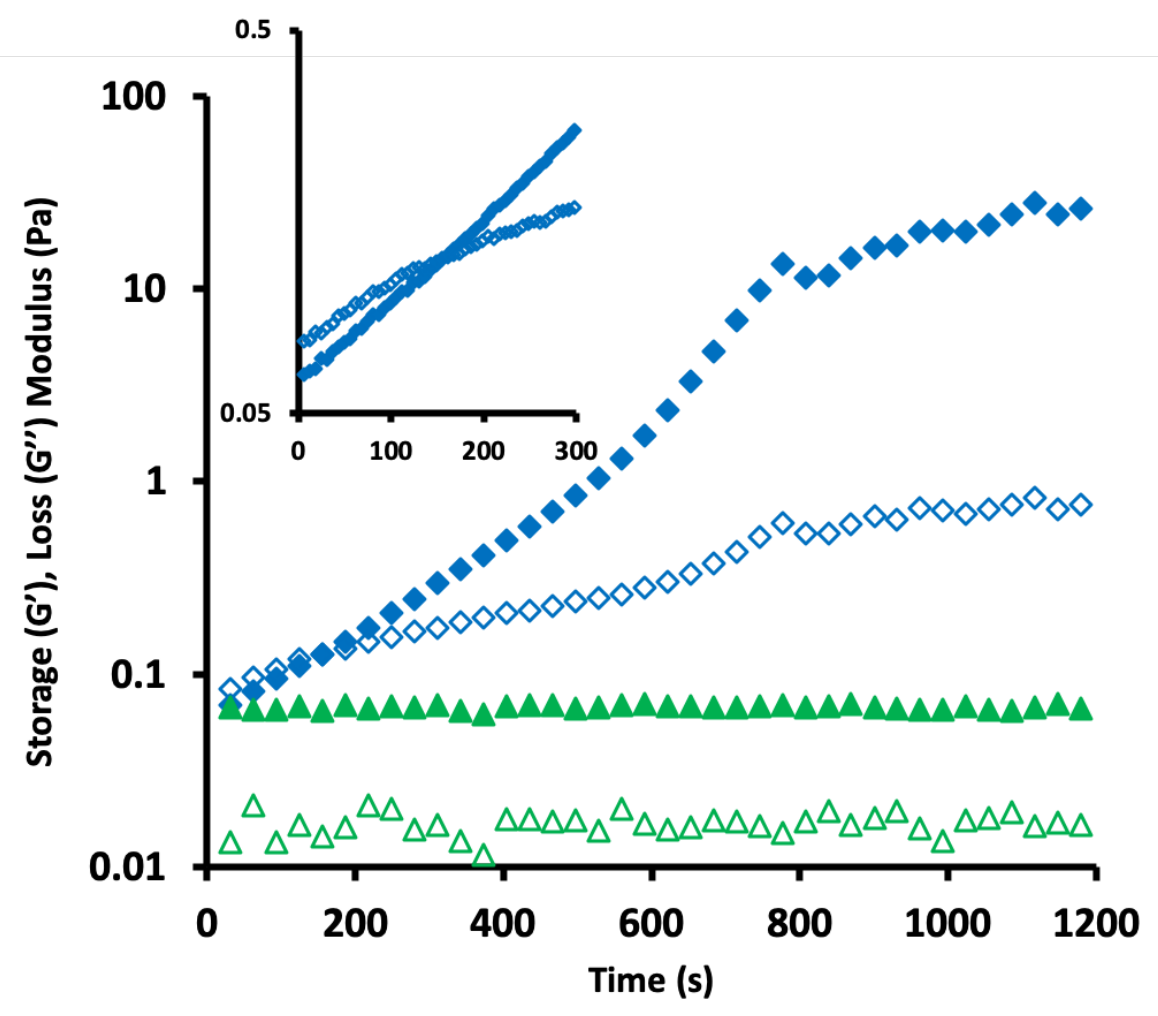

Figure S6. Evolution of storage (filled symbols) and loss (unfilled symbols) moduli for $3 \mathrm{wt} \%$ (blue diamonds) and $1 \mathrm{wt} \%$ (green triangles) CNC-POEGMA suspensions. Values presented are the average for 3 independent runs. 

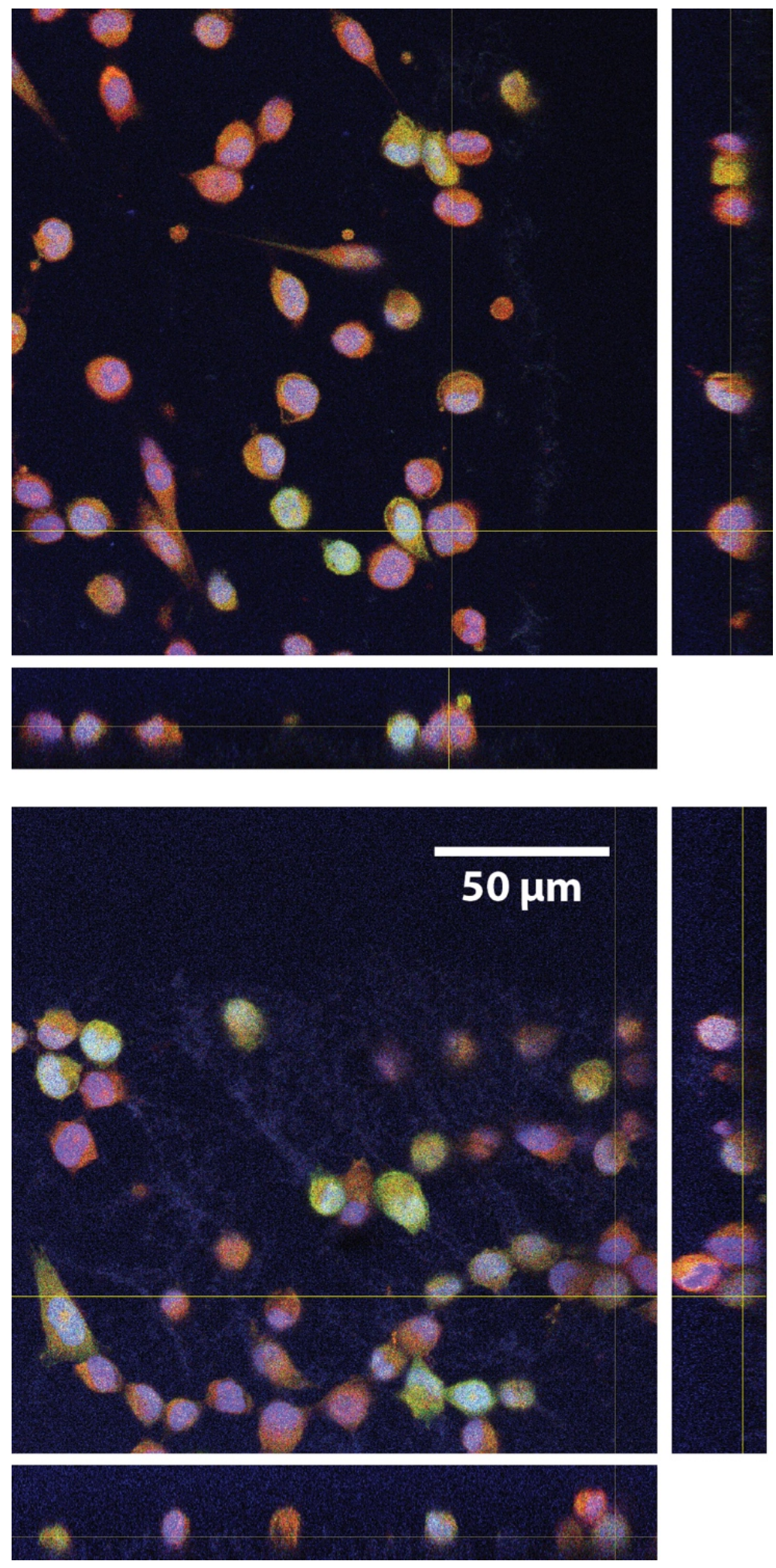

Figure S7. Orthogonal projections of confocal z-stack images for PC3 cells grown on CNCPOEGMA aerogels made using $7 \mu$ m-thick Parylene molds. 


\section{AUTHOR INFORMATION}

\section{Corresponding Author}

*Prof. Jose Moran-Mirabal, email: mirabj@mcmaster.ca

\section{Author Contributions}

The manuscript was written through contributions of all authors. All authors have given approval to the final version of the manuscript.

\section{Funding Sources}

Moran-Mirabal: Natural Sciences and Engineering Research Council Discovery Grant RGPIN 392283, Ontario Ministry of Research and Innovation Early Researcher Award, Canada Research Chairs, France Canada Research Fund - New Collaborations Program.

Cranston: Natural Sciences and Engineering Research Council Discovery Grant RGPIN 402329, Ontario Ministry of Research and Innovation Early Researcher Award, Canada Research Chairs.

Cerf: French National Research Agency Grant ANR-15-CE19-0020.

Hoare: Natural Sciences and Engineering Research Council Discovery Grant RGPIN 201706455, Canada Research Chairs. 